\title{
Stretched Polymers in a Poor Solvent
}

\author{
Peter Grassberger and Hsiao-Ping Hsu \\ John-von-Neumann Institute for Computing, Forschungszentrum Jülich, D-52425 Jülich, Germany
}

(October 23, 2018)

\begin{abstract}
Stretched polymers with attractive interaction are studied in two and three dimensions. They are described by biased self-avoiding random walks with nearest neighbour attraction. The bias corresponds to opposite forces applied to the first and last monomers. We show that both in $d=2$ and $d=3$ a phase transition occurs as this force is increased beyond a critical value, where the polymer changes from a collapsed globule to a stretched configuration. This transition is second order in $d=2$ and first order in $d=3$. For $d=2$ we predict the transition point quantitatively from properties of the unstretched polymer. This is not possible in $d=3$, but even there we can estimate the transition point precisely, and we can study the scaling at temperatures slightly below the collapse temperature of the unstretched polymer. We find very large finite size corrections which would make very difficult the estimate of the transition point from straightforward simulations.
\end{abstract}

\section{INTRODUCTION}

Deformations of polymers have been studied for many years, such as stretching a single DNA or titin macromolecule [1] 6]. Such experiments will become even more important with the rapid improvement in single molecule experiments [7]. Their theoretical understanding has attracted much attention. Most such experiments have been performed in good solvents, where also the theory is best understood [8]. But of particular interest in biology is the stretching of a collapsed polymer, i.e. of a polymer in a poor solvent below the $\Theta$-temperature. Up to now there seem to exist only few experiments in this regime [9]. Unfolding proteins in this way could e.g. give important information on their spontaneous folding pathways.

Although there exist several theoretical papers on stretching of collapsed polymers [10 16], we believe that further work is needed for a full understanding. It is generally believed that there is a first order transition between the globule and the stretched phases in 3 dimensions. But it seems that precise estimates of the critical force do not exist. Also, there are no predictions for the scaling laws expected when the temperature approaches the collapse temperature $T_{\theta}$ from below. Finally, it seems that there is only one paper [16] which deals with $d=2$. Moreover, while it is claimed in [16] that the transition is also first order in $d=2$, we shall find there a rather different situation and a second order transition.

A stretched polymer in a bad solvent is modeled as a biased interacting self-avoiding random walk (BISAW) on a regular lattice (square in $d=2$, simple cubic in $d=3$ ) with nearest neighbour attraction. In this model no two monomers can visit the same site. The attraction is taken into account by a Boltzmann factor $q=e^{-\beta \epsilon}$ for each pair of non-bonded monomers occupying nearest neighbours on the lattice. Here $\beta=1 / k T$ and $\epsilon<0$ is the attractive potential between non-bonded nearestneighbour pairs. The stretching is described by a factor $b=\exp (\beta a F)$ where $a$ is the lattice constant and $F$ is the stretching force [17]. The partition sum is therefore

$$
Z=\sum_{w a l k s} q^{m} b^{x}
$$

where $m$ is the number of non-bonded occupied nearestneighbour pairs, and $x$ is the distance (in units of lattice constants) between the two end points of the chain in the direction of $\mathbf{F}$. We are interested in polymers which form collapsed globules if they are not stretched, i.e. $q>q_{\theta}$ where $q_{\theta}=e^{-\epsilon / k T_{\theta}}$.

In the present paper we employ the Pruned-EnrichedRosenbluth Method (PERM) [18] to study the phase transition of the BISAW in $d=2$ and 3. But we shall see that, in order to understand the BISAW, we need also some other results. More precisely, we will need also some more results on the unbiased interacting selfavoiding random walk (ISAW), both on infinite and on finite lattices. On finite lattices with periodic boundary conditions we can study the bulk behaviour of collapsed polymeric matter without being disturbed by surface effects 19,18.

As we shall see, a stretched collapsed polymer in $d=2$ forms, in the infinite chain length limit $N \rightarrow \infty$ and for forces below the transition to the stretched phase, a compact object of a shape shown in Fig. 1. There the boundaries are circular arcs, the density inside is independent of the stretching force $F$, and the shape (i.e. the angles at the two extreme points) is determined both by $F$ and by $q$. The transition to the stretched phase occurs when these angles tend to zero.

In $d=3$ the situation is more complicated because of the Rayleigh instability. For $F<F_{c}$ one has an object of roughly elongated ellipsoidal shape, but for $F=F_{c}$ two phases coexist: Part of the chain is stretched, while the rest is still collapsed (Fig. 2). In the collapsed part, the density is the same as in an unstretched globule (for $N \rightarrow \infty)$. The stretched part might consist of a single piece (tadpole configuration [20]), or of two pieces as in Fig. 2. Within our theory both configurations have the same energies. The critical force $F_{c}$ vanishes linearly when $T \rightarrow T_{\theta}$. 
The paper is organized as follows: In Sec. II we treat the 2-dimensional case. We first formulate in more detail the model sketched above, and we show that it is in good agreement with simulations both of stretched and unstretched chains. The 3-dimensional case are discussed in Sec. III. Conclusions are drawn in Sec. IV. Finally, details of the Monte Carlo algorithm are given in an appendix.

(a)



(b)

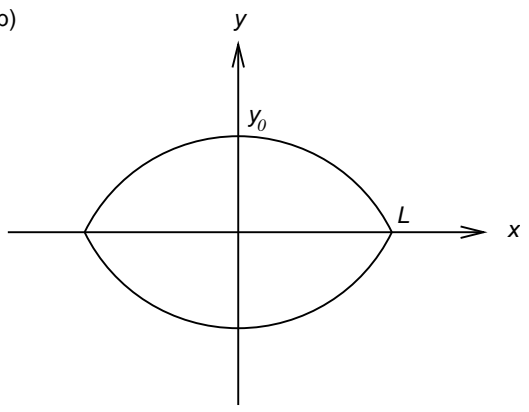

FIG. 1. (a) The geometric shape of a stretched polymer in a poor solvent in $d=2$, (b) Coordinates used in the calculation.

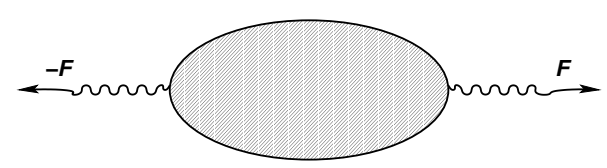

FIG. 2. Schematic drawing of a stretched polymer in a poor solvent in $d=3$, at an intermediate force $\mathbf{F}$ where the collapsed and extended phases coexist.

\section{II. $\mathrm{D}=\mathbf{2}$}

Let us first consider an unstretched polymer in $d$ dimensions, for any $d \geq 2$, below the $\Theta$-point. In the following, the lattice constant will always be $a=1$. For large $N$ and for infinite lattices the polymer forms a globule with a bulk monomer density $\rho(T)$ which tends to zero for $T \rightarrow T_{\theta}, \rho(T) \sim\left(T_{\theta}-T\right)^{\beta}$. For $d \geq 3$ we have $\beta=1$ and logarithmic corrections 18, but for $d=2$ the power $\beta$ seems to be unknown. For $T \rightarrow 0$ we have $\rho(T) \rightarrow 1$.
The free energy for such a polymer consists of two parts: An extensive bulk contribution $\propto N$, and a surface contribution $\propto N^{(d-1) / d}$. For $d=2$ this reads

$$
-\log Z_{N}(q, b=1) \approx \mu_{\infty}(q) N+\tilde{\sigma}(q) \sqrt{N}
$$

with $\mu_{\infty}$ being the chemical potential per monomer in an infinite chain, and $\tilde{\sigma}$ is related to the surface tension (=free energy per unit of perimeter length) $\sigma$ by

$$
\tilde{\sigma} \sqrt{N}=2 \pi R \sigma
$$

with $\pi R^{2} \rho=N$. Combining the last two equations we obtain

$$
\sigma=\frac{1}{2} \tilde{\sigma} \sqrt{\frac{\rho}{\pi}}
$$

Now assume we start stretching the polymer by pulling at the two ends. The first effect will be that the ends are drawn toward the surface and the whole globule is rotated such as to minimize the potential energy. We will neglect the change in entropy associated to that. When the stretching force increases, we develop a shape as indicated in Fig. 1a. We shall assume that the bulk is incompressible, so that we still have the same bulk free energy, and we obtain our final ansatz

$$
-\log Z_{N}(q, b) \approx \mu_{\infty} N+\sigma P-2 L F
$$

where $P$ is the perimeter and $2 L$ is the end-to-end distance. Parameterizing the shape by a function $y=y(x)$ with $y(0)=y_{o}, d y /\left.d x\right|_{x=0}=0$, and $y(L)=0$ (see Fig. 1b), we have

$$
P=4 \int_{0}^{L} d x \sqrt{1+(d y / d x)^{2}}, \quad 4 \rho \int_{0}^{L} d x y(x)=N .
$$

The optimal shape is obtained by maximizing $Z_{N}$ at constant $N$. The Euler equations for this maximization problem consist of two parts. The first, resulting from $\partial Z_{N}(q, b) / \partial L=0$, gives the equilibrium condition between stretching and surface tension forces,

$$
F=2 \sigma \cos \phi
$$

where the angle $\phi$ is indicated in Fig. 1a. Thus the critical point, corresponding to $\phi=0$, is at $F=2 \sigma$ or

$$
b_{c}=e^{2 \sigma} \text {. }
$$

The second part, maximization with respect to $y(x)$, gives that $y(x)$ is a circular arc. Using the angle $\phi$ indicated in Fig. 1a, we have then

$$
P=4 L \frac{\phi}{\sin \phi}, \quad N / \rho=2 L^{2} \frac{\phi-\sin \phi \cos \phi}{\sin ^{2} \phi} .
$$

For $b<b_{c}$ we have 


$$
-\log Z_{N}(q, b)=\mu_{\infty} N+\sigma N^{1 / 2}[8(\phi-\sin \phi \cos \phi) / \rho]^{1 / 2}
$$

with

$$
\phi=\arccos \frac{\log b}{2 \sigma} .
$$

Eqs. (8)-(11) form our final solution. They involve the three temperature dependent material constants $\mu_{\infty}, \sigma$, and $\rho$. The former two can be estimated from Eq. (2) if we measure in addition the gyration radius, but more precise estimates of $\mu_{\infty}$ and $\rho$ result from simulations on finite lattices in the dense limit [21, 18]. Thus our strategy in verifying the above theory numerically consists of the following steps:

1. We simulate chains on finite lattices of size $L \times L$ (typically with $L=8$ to 64 ) by means of the PERM algorithm which gives us directly estimates of the partition sum. For $q>q_{\theta}$ its logarithm is not convex. For suitably adjusted $\mu_{L}, \log Z_{N}(q, b=$ $1, L)+\mu_{L} N$ has two peaks of equal heights: One at $N \approx 0$, the other at $N \approx L^{2} \rho$. Extrapolating to $L=\infty$ we obtain $\mu_{\infty}$.

2. In the next step we simulate chains on (practically) infinite lattices, again by means of PERM. Using Eq. (2) and the already obtained values of $\mu_{\infty}$ and $\rho$ we then obtain $\sigma$.

3. Finally we simulate stretched polymers, to compare with the prediction of Eq. (10). In addition to $Z_{N}(q, b)$ we measure in these runs also $\langle x\rangle$ which should be equal to $2 L$ for large $N$ as long as $b<b_{c}$. We should, however, immediately warn that these latter measurements are not very conclusive, since finite size corrections are large in this regime. We might point out that for $b=1$ there is the exact result

$$
b \frac{\partial}{\partial b}\langle x\rangle=\left\langle x^{2}\right\rangle
$$

Since $\left\langle x^{2}\right\rangle \sim N$ for collapsed polymer in $d=2$, we thus have $\langle x\rangle \sim N$ for very small $b$. This shows that the above model (which would give $\langle x\rangle \sim N^{1 / 2}$ ) cannot be correct for small $N$, as we had indeed pointed out already before.

In Fig. 3 we show $\log Z_{N}+\mu_{L} N$ for $q=2.4, b=1$, and for finite lattices with $L^{2}=2^{7}, 2^{8}, \ldots, 2^{12}$ sites, plotted against $N / L^{2}$. The curves are in the same order as shown in the legend. Since $q_{\theta} \approx 1.95$ for this model [22], this is deep inside the collapsed regime. We used helical boundary conditions. When $L^{2}$ was not an integer, the effective lattice shape was not a perfect square, but this led to negligible corrections. The values of $\mu_{L} N$ were fixed by demanding the right hand peak to have height exactly zero. The values obtained this way are shown in Fig. 4. They are plotted there against $L^{-1.72}$ because this gave the best straight extrapolation to $L=\infty$. The extrapolated value is $\mu_{\infty}=-1.3213(1)$. The positions of the right hand peaks give finite lattice approximations to the density $\rho$. Extrapolating to $L=\infty$ gave $\rho=0.84(1)$. Analogous simulations were also done at different values of $q$.

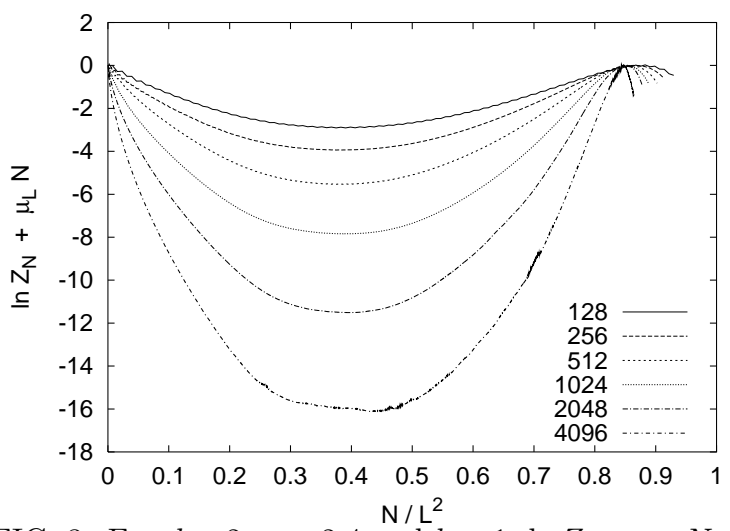

FIG. 3. For $d=2, q=2.4$ and $b=1, \ln Z_{N}+\mu_{L} N$ versus $N / L^{2}$ for finite lattices with $L^{2}=2^{7}, 2^{8}, \ldots, 2^{12}$. The values of $\mu_{L}$ were fixed by demanding that the peaks at $N / L^{2} \approx 0.85$ have zero height.

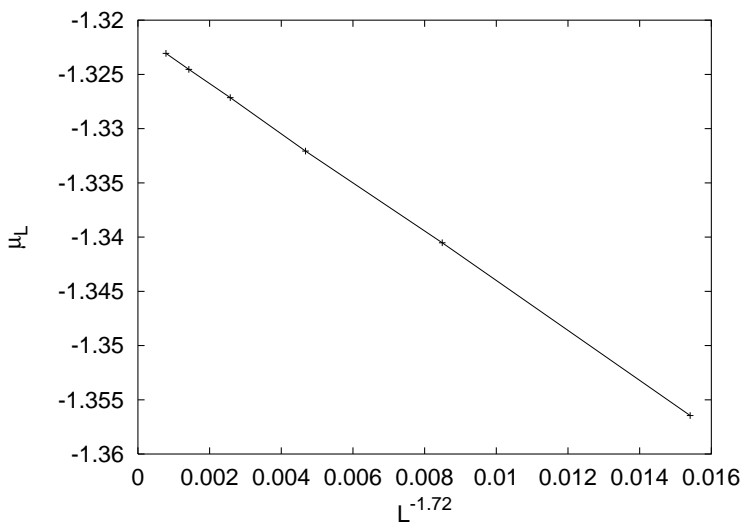

FIG. 4. $\mu_{L}$ as obtained from Fig. 3, plotted against $L^{-1.72}$ which gave the most straight extrapolation. Error bars are ca. 0.00005 , smaller than the symbol sizes. The extrapolation to $L=\infty$ is $\mu_{\infty}=-1.3213 \pm 0.0001$.

Results from unbiased ISAW on an 'infinite' lattice (i.e. on a lattice that was so large that no walk reached the boundary) are shown in Fig. 5. There we plot $\log Z_{N}+\mu_{\infty} N$ against $N^{1 / 2}$. From Eq. (2) we expect this to give a straight line with slope $-\tilde{\sigma}$. Actually the line is slightly curved, indicating that there are further finite size corrections for small $N$ and systematic sampling 
corrections at large $N$. The latter are indeed to be expected. When we constructed histograms of tour weights (see the appendix), we found that the simulations are unreliable for $N>1000$. We should have shown only the data for $N<1000$, but we showed all data, for the following reasons:

- we want to compare these simulations with biased and finite volume simulations of the same length which are easier for PERM and which are thus still reliable for $N=3500$, the longest chains used in Fig. 5.

- Even if we cannot be sure that the data for $N>$ 1000 are correct, we cannot argue either that they must be wrong. They are most likely too low, since PERM has difficulties to sample configurations which start out (as the chain grows) to have high energy (i.e. few contacts), but which become 'good' during later growth stages. But we do not know how important this is.

The curvature observed in Fig. 5 dominates the error in our estimate $\tilde{\sigma}=0.46(2)$. Combining this with the previous estimate for $\rho$ we obtain $\sigma=0.119(5)$, and from this we predict $b_{c}=1.269(13)$. Remember that all this is for $q=2.4$.

Finally, results from simulations with $b \geq 1$ are shown in Figs. 6 to 9. In Fig. 6 we show $\log Z_{N}(q, b)+\mu_{\infty}(q) N$ versus $N$. We expect these curves to become horizontal for $N \rightarrow \infty$ as long as $b \leq b_{c}$, while they should increase linearly for $b>b_{c}$. This is indeed seen, although we now find a slightly larger value $b_{c}=1.285(6)$. In spite of the small discrepancy with the predicted value we consider this as remarkable agreement. Our data are not precise enough to allow a comparison with the detailed predictions for $1<b<b_{c}$.

For $b>b_{c}$ the chemical potentials $\mu_{\infty}^{(s)}(q, b)$ in the stretched phase can be estimated easily by demanding that $\log Z_{N}(q, b)+\mu_{\infty}^{(s)}(q, b) N$ becomes $N$-independent for large $N$. The results are shown in Fig. 7. Although this figure clearly shows a continuous transition, the data are not precise enough to quote a meaningful critical exponent. It is compatible with $3 / 2$, but the uncertainty is large.

Among the other measurements during these runs, the most interesting are those of $\langle x\rangle$. Again they are not precise enough for a detailed comparison with the predictions in the regime $b<b_{c}$. But they also show clearly the phase transition at $b=b_{c}$, since $\langle x\rangle$ rises linearly with $N$ only in the stretched phase (see Fig. 8). Values of $v=d\langle x\rangle / d N$, obtained by extrapolating the observed slopes in plots of $\langle x\rangle$ against $N$ toward $N=\infty$, are shown in Fig. 9. Again we see a continuous transition with an exponent which is roughly equal to 0.7 , but uncertainties are too large to make a more definite statement.



FIG. 5. Values of $\ln Z_{N}(q, b=1)+\mu_{\infty} N$ versus $N^{1 / 2}$ for $d=2, q=2.4$, and $\mu_{\infty}=-1.3213$. The dashed line has slope $-\tilde{\sigma}(\tilde{\sigma}=0.46 \pm 0.02)$.

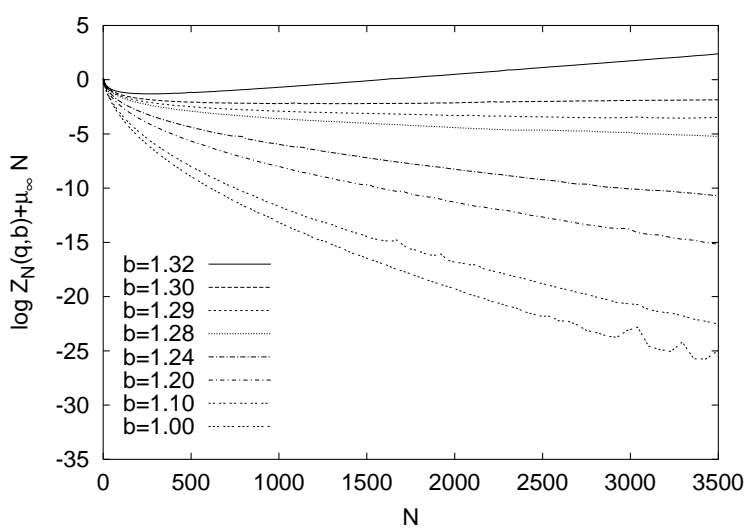

FIG. 6. Values of $\ln Z_{N}(q, b)+\mu_{\infty} N$ versus $N$ for the same $q$ and $\mu_{\infty}$ as in Fig. 5, and for various values of $b$.

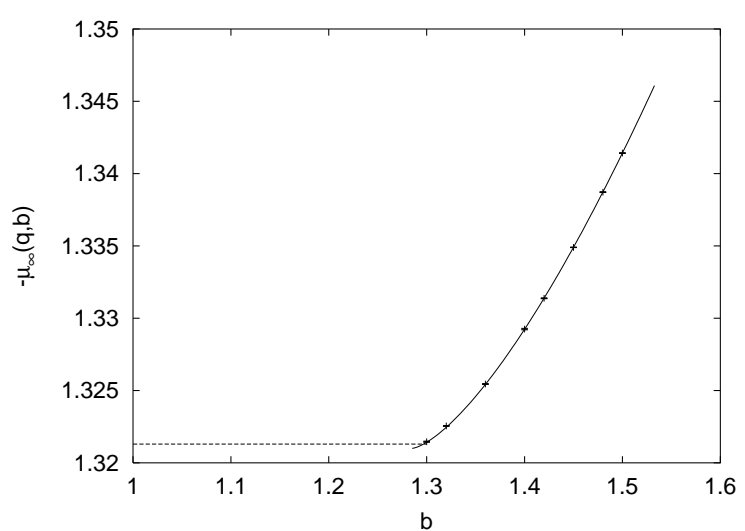

FIG. 7. Values of $\lim _{N \rightarrow \infty}\left[N^{-1} \ln Z_{N}(q, b)\right]$ versus $b$, for $d=2$ and $q=2.4$. For $b>b_{c}$ this is $-\mu_{\infty}^{(s)}(q, b)$. For $b<b_{c}(\approx 1.3)$, we replaced the actual numerical estimates by the theoretical value $-\mu_{\infty}(q)$. 


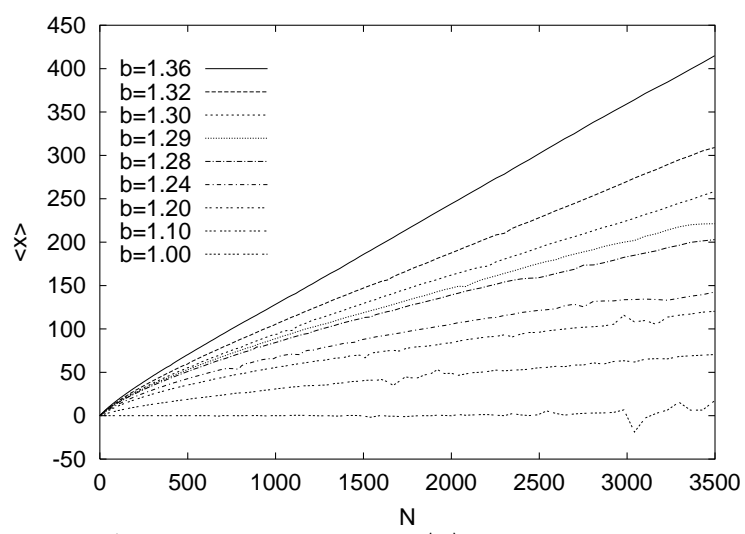

FIG. 8. Average displacement $\langle x\rangle$ in the bias direction for $d=2, q=2.4$ and for various values of $b$.



FIG. 9. Asymptotic "velocity" $d\langle x\rangle / d N$ in the bias direction for $d=2, q=2.4$ and for various values of $b$.

Before leaving this section we should remark that we checked carefully, in view of Ref. [16], that the unfolding transition is indeed second order in our model. We looked e.g. at histograms analogous to those in Figs. 12 and 13 . We definitely saw no hint of bistability or hysteresis. Together with the good agreement with the above model this seems definitely to rule out a first order transition. We cannot rule out, however, that the transition is first order in another microscopic model such as the bond fluctuation model studied in [16.

\section{III. $\mathrm{D}=3$}

Some of the treatment for $d=2$ can be carried over to 3 dimensions with minor modifications. Instead of Eq. (2) we now have 23, 19]

$$
-\log Z_{N}(q, b=1) \approx \mu_{\infty}(q) N+\tilde{\sigma}(q) N^{2 / 3} .
$$

Again we estimate $\mu_{\infty}$ most reliably from finite lattice simulations in the dense limit [18]. Again $\tilde{\sigma}$ can be related to a surface tension, and can be estimated numerically by plotting $\log Z_{N}(q, b=1)+\mu_{\infty}(q) N$ against $N^{2 / 3}$.

In the weak stretching regime we could still try to solve the optimal shape, but this time the minimization problem is more complicated and we were not able to find an explicit solution. But more important, we do not expect a continuous transition as in $d=2$. The reason is that we expect a Rayleigh instability when the globule is stretched too much, which is expected to occur, before the limit $\phi=0$ is reached. Thus we cannot expect to be able to predict the transition point as in $d=2$.

On the other hand, things also simplify because the critical exponents at the $\Theta$ point now should be mean field like (the upper critical dimension for the $\Theta$ point is 3 [8]). This means in particular that $\tilde{\sigma}, \rho$ and $\mu_{\infty}(q)-$ $\mu_{\infty}\left(q_{c}\right)$ all should vanish as $\sim\left(T_{\theta}-T\right)$ when $T \rightarrow T_{\theta}$ from below.

For a stretched globule with $b<b_{c}$ we expect that $Z_{N}$ differs from the value for $b=1$ only by its changed surface, i.e. by terms $\sim N^{2 / 3}$. For $b>b_{c}$ we have a string-like phase whose free energy should increase linearly with $N$ with very small corrections,

$$
-\log Z_{N}\left(q, b>b_{c}\right) \approx \mu^{(s)}(q, b) N,
$$

where the chemical potential $\mu^{(s)}(q, b)$ in the stretched phase is a function independent of the chemical potential $\mu_{\infty}(q, b)$ in the globular phase. The end-to-end distance in the stretched phase is given by $\langle x\rangle=N v(q, b)$ with

$$
v(q, b)=-b \frac{\partial \mu^{(s)}(q, b)}{\partial b} .
$$

Stability of the stretched phase requires that $\partial v(q, b) / \partial b>0$, i.e.

$$
b \frac{\partial \mu^{(s)}(q, b)}{\partial b}, \quad\left(b \frac{\partial}{\partial b}\right)^{2} \mu^{(s)}(q, b)<0 .
$$

In between these two regimes we expect a coexistence region where part of the chain forms a (single) globule, while the rest forms one or two stretched pieces. A somewhat crude model of a polymer in the coexistence region which, however, catches all essential features including finite size effects, is the following: The stretched part has $N_{s}$ monomers, and its partition sum is described by Eq. (14) with $N$ replaced simply by $N_{s}$. The globular part has $N_{g}=N-N_{s}$ monomers, and its partition sum is described by Eq. (13) with $N$ replaced by $N_{g}$. The total partition sum is just the product of the two, i.e. the free energy is just the sum of free energies of the two parts. Notice that this involves a number of approximations:

- There is no penalty for the area where the globule and the stretched part(s) are attached to each other. Such a free energy contribution should be independent of $N$ and can be safely neglected. 
- The globule is approximated by a sphere. This is a more serious approximation. It systematically overestimates the free energy, by an amount $\propto N_{g}^{2 / 3}$. This is of the same order of magnitude as if a wrong surface tension were used. It should therefore lead to quantitative errors, but not to qualitative ones. Moreover, the errors should be small because too elongated globules are prevented by the Rayleigh instability.

- We neglect all fluctuations.

The total free energy in the coexistence region is thus, for a fixed total end-to-end distance $x$ (and not denoting explicitly the dependence on $q$ ),

$$
-\log Z_{N}=N_{g} \mu_{\infty}+N_{g}^{2 / 3} \tilde{\sigma}+\left(N-N_{g}\right) \mu^{(s)}(b)-x F .
$$

Minimizing this with respect to $N_{g}$ we obtain

$$
\mu_{\infty}-\mu^{(s)}(b)+\frac{2 \tilde{\sigma}}{3} N_{g}^{-1 / 3}=0 .
$$

This can be read as an implicit equation for $b$. If solved, it gives $b$ as a function of $N_{g}$. Notice that $b$ is independent of $N_{s}$ : According to our model the force needed to pull out the chain from the globule depends on its size through the surface tension (last term in Eq. (18)), but not on the length $N_{s}$ of the stretched part.

In the limit $N_{g} \rightarrow \infty$ this term does not contribute, and we obtain the condition for the true transition point

$$
\mu^{(s)}\left(b_{c}\right)=\mu_{\infty} .
$$

For finite $N_{g}$ we see that at the coexistence point $\mu^{(s)}(b)$ decreases with $N_{g}$. Since $\partial \mu^{(s)}(b) / \partial b<0$, this implies that the effective critical $b$ increases with $N_{g}$. In other words, as the chain is pulled out from the globule, $N_{g}$ shrinks, and thus the force needed to pull out more of the chain decreases. This is the basic instability which makes the transition first order and shows that our ansatz is consistent. It implies that a finite globule will be entirely pulled open as soon as $b>b_{c}-$ const $/ N^{1 / 3}$. Notice that a more realistic model (where the spherical globule is replaced by some other shape with limited aspect ratio) would still give the same qualitative results: For infinitely large globules, the critical point is given by equating $\mu^{(s)}\left(b_{c}\right)$ with the chemical potential for unstretched globules; and finite size corrections to this are negative and decrease as $N^{-1 / 3}$.

In a first set of simulations we determined numerically $\mu_{\infty}(q)$ and $\rho(q)$ for a wide range of $q$. We did this again, as in $d=2$, by performing the simulations on finite lattices in the dense limit. In a second step, we obtained $\tilde{\sigma}$ from simulations of unbiased ISAWs in infinite lattices. In contrast to $d=2$, here we encountered the problem that there are significant further corrections to the asymptotic ansatz Eq. (13). We illustrate this in Fig. 10 where we plot $\log \left[Z_{N-n}(q) / Z_{N+n}(q)\right] /(2 n)$ against $N^{-1 / 3}$ for $b=1$ and several values of $q$. Here, $n=1+\lfloor N / 20\rfloor$. The points on the $y$-axis are obtained from dense limit simulations. The straight lines are extrapolations constrained to pass through these points. Their slopes are $2 \tilde{\sigma} / 3$. While the curves are compatible with these extrapolations, close inspection shows that they are not convex as one might have guessed naively. Thus, extrapolations not aided by the dense limit simulations would be prone to large errors.

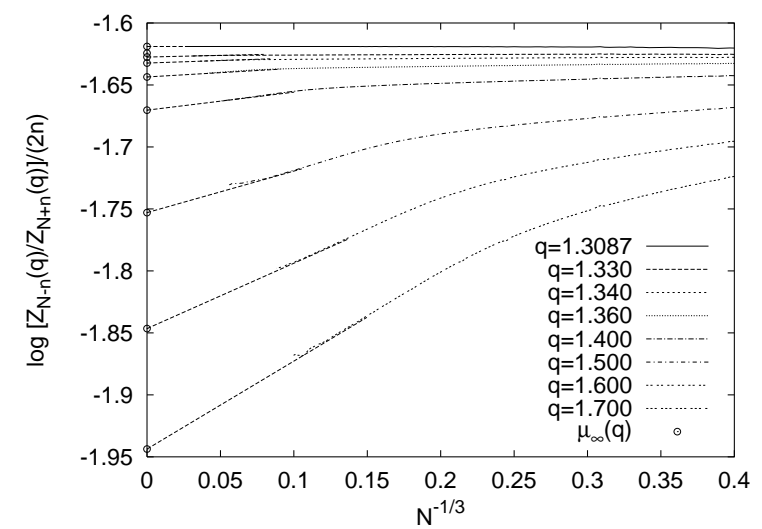

FIG. 10. $\ln \left[Z_{N-n}(q) / Z_{N+n}(q)\right] /(2 n)$ versus $N^{-1 / 3}$ for $d=3, b=1$ and several values of $q$. The points on the $y$-axis are obtained from dense limit simulations on finite lattices, and the straight lines are extrapolations to $N \rightarrow \infty$, constrained to pass through these points.

Finally, we performed simulations of BISAW (on 'infinite' lattices). We shall discuss in detail only the most extensive simulations, done at $q=1.5$ which is deep in the collapsed region $\left(q_{\Theta}=1.3087(3)\right.$ for this model [18]). But similar simulations were also done at different values of $q$.

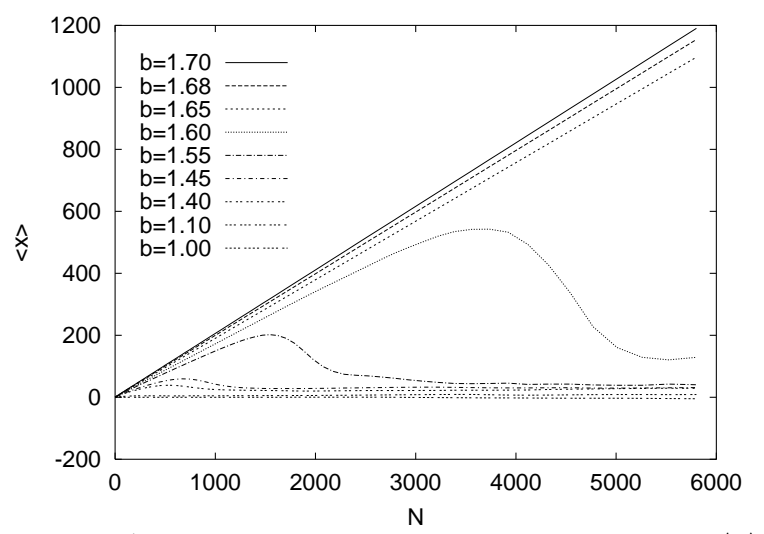

FIG. 11. Average displacement in the bias direction $\langle x\rangle$ for $d=3, q=1.5$ and for various values of $b$. 
Average displacements $\langle x\rangle$ against $N$ for various biases $b$ are shown in Fig. 11. For $b=1$, we have of course $\langle x\rangle=0$. With increasing $b,\langle x\rangle$ still remains close to zero for large $N$ and increases very slowly as $N \rightarrow \infty$ as long as $b$ is close to 1 . But for small $N$ it increases roughly $\propto N$, with an abrupt decrease in a narrow range which shifts toward larger $N$ when $b$ is increased. As $b$ increases above a certain value $b_{c}$, the linear range of $\langle x\rangle$ versus $N$ extends to $N=\infty$, indicating the phase transition from the collapsed to the stretched polymer phase. From Fig. 11 the critical point of the finite system might be guessed to be between 1.60 and 1.65 , but we will see that finite-size corrections are very large and $b_{c}$ is actually larger than 1.85 .

Very similar behaviour is seen for $R_{N}^{2}$ and for the average number of non-bonded nearest-neighbour pairs $\langle m\rangle$ (not shown). For $b>b_{c}$ they increase linearly with $N$, $R_{N}^{2} \propto\langle m\rangle \propto N$. For $b<b_{c}$ but close to $b_{c}$ they first show the same behaviour, indicating that short polymers would be stretched at this $b$, but then cross over to the collapsed phase in a very narrow range of $N$.

We claim that this is a first (yet inconclusive) indication for the first order nature of the transition. More direct indications are obtained by studying the histograms of $x$ and $m$. The histograms shown in the following figures are obtained by combining data from runs with different $q$ and $b$ and reweighting them. Combining MC results from different temperatures is not trivial for conventional Metropolis-type Monte Carlo algorithms where absolute normalization is unknown [24]. In contrast, it is straightforward for PERM, since PERM gives directly estimates of the partition sum and of the properly normalized histograms

$$
P_{q, b}(m, x)=\sum_{w a l k s} q^{m^{\prime}} b^{x^{\prime}} \delta_{m, m^{\prime}} \delta_{x, x^{\prime}}
$$

Reweighting histograms obtained with runs performed nominally at $q$ and $b$ is trivially done by

$$
P_{q^{\prime}, b^{\prime}}(m, x)=P_{q, b}(m, x)\left(q^{\prime} / q\right)^{m}\left(b^{\prime} / b\right)^{x} .
$$

Combining results from different runs can then be either done by selecting for each $(m, x)$ just the run which produced the least noisy data (which was done here in most cases), or by assuming that the statistical weights of different runs are proportional to the number of 'tours' 18] which contributed to $P_{q, b}(m, x)$.

In the present work we studied only single variable histograms $P_{q}(m)$ and $P_{b}(x)$, for which the above holds with the appropriate modifications.

Histograms of $m$ for fixed $q$ and $b$, and for three different values of $N$, are shown in Fig. 12. For $N=1500$ and $N=2000$ we see two peaks, corresponding to the collapsed (right hand, $m \approx 0.9 N$ ) and stretched (left hand, $m \approx 0.4 N$ ) phases. For $N>2000$ all chains would be collapsed, while for $N \leq 1000$ all chains are stretched, in agreement with Fig. 11.



FIG. 12. Histograms of the number of non-bonded nearest-neighbour pairs $P(m)$ versus $m / N$ for $d=3, q=1.5$, and $b=1.55<b_{c}$. The peaks near $m / N \approx 0.4$ correspond to the stretched phase, the ones near $m / N \approx 0.9$ to the collapsed phase. Notice that chains with $N=1000$ are for these values of $b$ and $q$ entirely in the stretched phase (there is no peak near $m / N \approx 0.9$ ), in agreement with Fig. 11. Normalization is arbitrary.

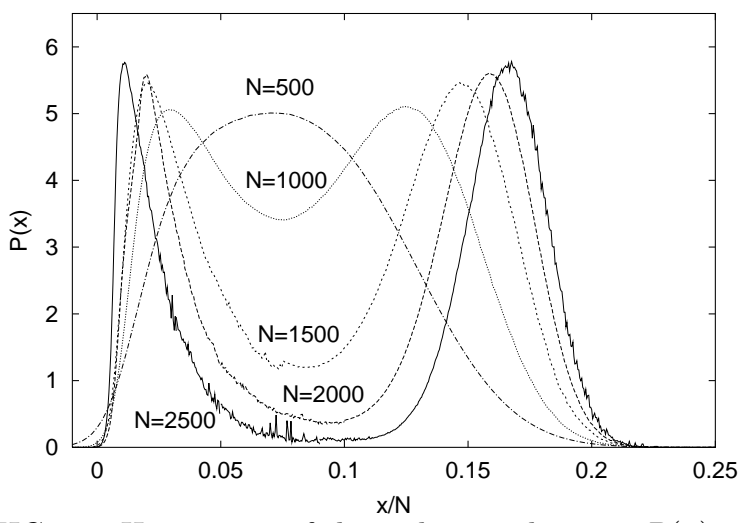

FIG. 13. Histograms of the end point distance $P(x)$ versus $x / N$ for $q=1.5$. Biases were adjusted so that both peaks have equal height: $b=1.4040(N=500), 1.4925(N=1000)$, $1.5386(N=1500), 1.5658(N=2000), 1.5855(N=2500)$. Normalization is arbitrary. The peak at $x / N \approx 0$ corresponds to the collapsed phase, the other to the stretched phase.

Analogous histograms of $x$ are shown in Fig. 13 for a wider range of $N$. We now see an even more pronounced double peak structure, with the left (right) peaks corresponding to the collapsed (stretched) phase. In this figure we kept $q=1.5$ fixed but varied $b$, so that both peaks have the same height for each $N$. In addition we adjusted the normalization arbitrarily such that all peaks have similar heights. We see clearly that the height of the minimum between the peaks shrinks to zero for $N \rightarrow \infty$, and that the horizontal distance between the peaks increases with $N$. Taken together, they form a clear indication for a first order transition. Notice that a double peak 
structure with decreasing minimum alone would not be a conclusive proof, as shown by the $\Theta$-point in dimensions $d \geq 4$ [25].

The values $b=b_{c}(N)$ for which the two peaks to have equal height (indicated in the caption of Fig. 13) are effective finite $N$ transition points. According to our phenomenological model we expect them to scale as

$$
\left|b_{c}(N)-b_{c}\right| \propto N^{-1 / 3} \text {. }
$$

The values obtained from Fig. 13 are plotted in Fig. 14 against $N^{-1 / 3}$. From Eq. (22) we expect them to fall onto a straight line. This is indeed the case, and the extrapolation to $N \rightarrow \infty$ gives $b_{c}=1.84 \pm 0.04$.



FIG. 14. Effective transition points $b_{c}(N)$ versus $N^{-1 / 3}$ for $d=3, q=1.5$. The true transition point $b_{c}=1.84 \pm 0.04$ is determined by extrapolating to the $y$-axis.

This estimate of $b_{c}$ is already more precise than any estimate we could obtain from Fig. 11. But an even more precise estimate is obtained by analyzing the partition sum itself. In Fig. 15 we show $\log Z_{N}(q, b)+\mu_{\infty} N$ versus $N$. For small $b$ the curves are close to the curve for $b=1$. As $b$ increases, the initial (small- $N$ ) parts of these curves are straight lines with less and less negative slopes. In this regime the polymer is stretched. As long as these slopes are negative, the straight lines will intersect the curve for $b=1$ at some finite value of $N$, say $N_{c}(b)$. Obviously these are the chain lengths where $b=b_{c}\left(N_{c}\right)$ (see Fig. 14).

For $N>N_{c}(b)$, the values of $\log Z_{N}(q, b)+\mu_{\infty} N$ must deviate from the straight lines. Indeed, the curves in Fig. 15 cannot cross each other since 26.

$$
\begin{aligned}
\log Z_{N}(q, b) & -\log Z_{N}(q, b=1) \\
& =\log \left[\langle\cosh (x \log b)\rangle_{b=1}\right] \\
& \geq \log \left[1+\frac{(\log b)^{2}}{2}\left\langle x^{2}\right\rangle_{b=1}\right] .
\end{aligned}
$$

In the simulations, such a crossing can of course happen due to metastability of the stretched phase. With our algorithm, the collapsed state has much lower energy close to the transition point, but also much lower entropy, so that it can easily be missed during a run with finite CPU time. Whenever this happened, were plotted in Fig. 15 the value predicted by Eq. (23) instead of using the direct estimate of $Z_{N}(q, b)$.

Since the curve for $b=1$ becomes horizontal for $N \rightarrow \infty$, the true phase transition occurs at that value of $b$ for which the straight line in Fig. 15 is also horizontal. This can be estimated very easily and with high precision, giving for $q=1.5$ our final estimate $b_{c} \approx 1.856(1)$. This is in perfect agreement with the above finite- $N$ extrapolation. Its error is dominated by the uncertainty of $\mu_{\infty}$

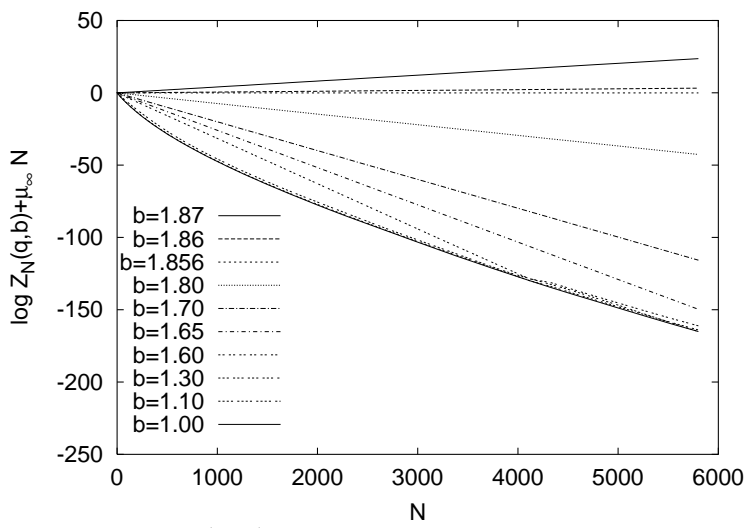

FIG. 15. $\ln Z_{N}(q, b)+\mu_{\infty} N$ for $d=3, q=1.5$, and for various values of $b$. The value $\mu_{\infty}=-1.7530 \pm 0.0003$ used in this plot was obtained from dense limit simulations on finite lattices.

The results of $\log \left(b_{c}(q)\right)$ and $\tilde{\sigma}(q)$ for a wide range of $q$ values are shown in Fig. 16. We see that both curves are roughly linear near the $\Theta$ point, with slopes close to one. Exact scaling laws $\log \left(b_{c}(q)\right) \sim q-q_{\theta}$ and $\tilde{\sigma}(q) \sim q-q_{\theta}$ cannot be expected because of the strong logarithmic corrections at the $\Theta$ point 19,18$]$.

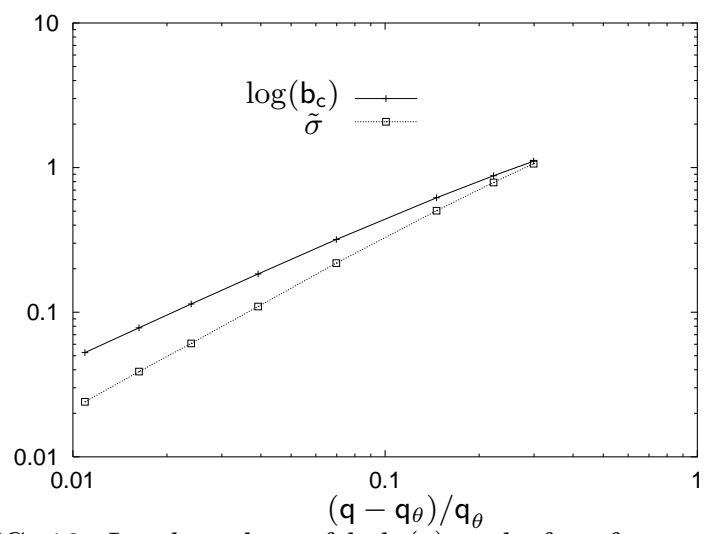

FIG. 16. Log-log plots of $\ln b_{c}(q)$ and of surface tensions $\tilde{\sigma}(q)$ versus $\left(q-q_{\theta}\right) / q_{\theta}$ for $d=3$. The continuous lines are drawn to guide the eye. 


\section{CONCLUSION}

By employing the PERM algorithm to the BISAW model with attractive interaction on square and simple cubic lattices, we have studied the process of stretching collapsed polymers in two and three dimensions. In $d=2$ we find a second order transition, in contrast to previous results for the bond fluctuation model [27]. We do not know whether this is due to an inherent difference in the models. In $d=3$ a clear first-order phase transition is observed, in agreement with all previous studies. But it seems that the present study is the first with a detailed study of the transition region and of finite size effects.

We indeed found the latter to be extremely important, in particular for $d=3$. Estimates of the critical force obtained without careful extrapolation to $N \rightarrow \infty$ would be grossly wrong. This is similar to the $\Theta$ collapse of unstretched polymers in high dimensions 25 where these finite size effects even mimicked a first order transition, while the true transition is second order. We believe that we can exclude the latter for the present case.

The most precise estimates of critical forces were obtained via a direct comparison of the free energies in the two phases. This is easily done with PERM, in contrast to most other Monte Carlo methods. We found that the partition sum of the collapsed phase is very closely related to that of completely unstretched collapsed polymers. An essential part of our numerical effort went indeed into improved estimates of the latter. In particular, we verified that the concept of a surface tension applies both to 2-d and 3-d collapsed polymers, and for $d=3$ we verified its scaling with temperature.

We compared the finite $N$ corrections in detail with phenomenological models which yield different order transitions in $d=2$ and $d=3$, and found perfect agreement. The latter is a bit surprising since fluctuations and surface layer thicknesses are neglected in the latter. These approximations should become exact in the limit of chain length $N \rightarrow \infty$, but our present chains, with $N \approx 10^{3}-10^{4}$, could be expected a priori to be much too short for this.

\section{APPENDIX}

PERM is a particular depth-first implementation of sequential importance sampling with reweighting 28. Polymer chains are built like random walks by adding one monomer at each step.

As in any such algorithm we have the freedom to sample these steps from a wide range of possible distributions, provided this additional bias is taken into account by suitable weight factors. First of all, we used a Rosenbluth like bias in avoiding steps which would lead to self intersections. Among the other possible steps we selected those parallel, antiparallel, and transverse to $\mathbf{F}$ with probabilities $p_{+x}: p_{-x}: p_{\perp}=\sqrt{b}: \sqrt{1 / b}: 1$. The Boltzmann factors for the pair interactions were taken into account entirely by the weight factors and did not enter into the step probabilities. Let us define $p_{\perp}^{(0)}=1, p_{ \pm}^{(0)}=b^{ \pm 1 / 2}$. Then we have $p_{i}=0$ if step $i$ is forbidden, and

$$
p_{i}=\frac{p_{i}^{(0)}}{\sum_{\text {allowed } j} p_{j}^{(0)}}
$$

else. The corresponding weight factors are then

$$
w_{i}=\frac{q^{m_{n}} b^{\Delta x_{i}}}{p_{i}} \quad\left(\Delta x_{i}=0,1, \text { or }-1\right),
$$

where $m_{n}$ is the number of neighbours of the new site already occupied by non-bonded monomers.

The total weight of a chain of length $n$ is then $W_{n}=$ $\prod_{n^{\prime} \leq n} w_{i_{n^{\prime}}}$. Every time an $n$-th monomer is added to the chain, we update the current estimate of the partition sum to

$$
\hat{Z}_{n}=M_{n}^{-1} \sum_{\alpha=1}^{M_{n}} W_{n}(\alpha),
$$

where $M_{n}$ is the number of chains reaching length $n$ and $W_{n}(\alpha)$ is the weight of the $\alpha$ th chain. Chains are cloned and pruned if their weight is above $3 \hat{Z}_{n}$ and below $\hat{Z}_{n} / 3$, respectively.

For different sets of simulations we measured different observables. In the dense limit simulations, e.g., we measured only the partition sum, while the largest number of observables was measured for stretched chains on infinite lattices. There we measured the partition sum $Z_{n}$, the average end-to-end displacement $\langle x\rangle$ parallel to the force, the average squared end-to-end distance $<R^{2}>$, and the number of contacts between non-bonded monomers $<m>$ which is a measure for the internal energy. We also measured histograms of the parallel displacement and of the contact number. In addition during all runs we made also some technical control measurements. Most importantly, we generated histograms of tour weight distributions [29] in order to test whether the results are statistically reliable or not.

In all cases we used a single integer to label lattice sites, and used helical boundary conditions. If the lattice size was a power of $2^{d}$, say $2^{k d}$ with integer $k$, then the neighbours of site $i$ are $i \pm 1, i \pm 2^{k}, \ldots i \pm 2^{(d-1) k}$, and integers outside the range $\left[0, \ldots 2^{k d}-1\right]$ are brought back to this interval by means of bitwise AND with $2^{k d}-1$. If the lattice size was a different power of 2 , say $2^{p}$, the numbers $1,2^{k}, \ldots 2^{(d-1) k}$ are replaced by the integers nearest to $1,2^{p / d}, \ldots 2^{(d-1) p / d}$.

In $d=2$ and for finite lattices, self avoidance and contacts were simply checked by means of bit maps: in an array of characters, each occupied site was marked ' 1 ', 
while each empty site was marked ' 0 '. For opens systems in $d=3$ this would have needed too much storage and we used hashing. In this way we could implement effectively infinite lattices with relatively small computer memory.

Acknowledgements: We thank Dr. Walter Nadler for very helpful discussions.

[1] T. T. Perkins, S. R. Quake, Q. E. Smith, and S. Chu, Science 264, 822 (1994).

[2] T. T. Perkins, D. E. Smith, R. G. Larson, and S. Chu, Science 268, 83 (1995).

[3] C. Bustamante, J. F. Marko, E. D. Siggia, and S. Smith, Science 265, 1599 (1994).

[4] M. S. Z. Kellermayer, S. B. Smith, H. L. Granzier, and C. Bustamante, Science 276, 1112 (1997).

[5] M. Rief, F. Oesterhelt, B. Heymann, and H. E. Gaub, Science 275, 1295 (1997).

[6] L. Tskhovrebova, J. Trinik, J. A. Sleep, and R. M. Simmons, Nature (London) 387, 308 (1997).

[7] T. Strick, J.-F. Allemand, V. Croquette, and D. Bensimon, Physics Today, October 2001, page 46 (2001)

[8] P.G. de Gennes, Scaling Concepts in Polymer Physics (Cornell Univ. Press, Ithaca, 1979)

[9] B.J. Haupt, to be published

[10] P.-Y. Lai, Physica A 221, 233 (1995).

[11] P.-Y. Lai, Phys. Rev. E 53, 3819 (1996).

[12] A. Halperin and E. B. Zhulina, Europhys. Lett. 15, 417 (1991).

[13] A. Halperin and E. B. Zhulina, Macromolecules 24, 5393
(1991).

[14] P.-Y. Lai, Phys. Rev. E 58, 6222 (1998).

[15] D. Göritz, S. Kreitmeier, and M. Wittkop, J. Macromol. Sci., Phys. B35, 615 (1996); M. Wittkop, S. Kreitmeier, and D. Göritz, Phys. Rev. E 53, 838 (1996); S. Kreitmeier, M. Wittkop, and D. Göritz, Phys. Rev. E 59, 1982 (1999).

[16] M. Wittkop, S. Kreitmeier, and D. G'oritz, Comput. Polym. Sci., 5, 187 (1995).

[17] Notice that we describe here polymers in equilibrium. A real polymer, when stretched from a random globule state, is not in equilibrium since knots cannot unfold during and after the stretching, unless we deal with phantom polymers.

[18] P. Grassberger, Phys. Rev. E 56, 3682 (1997).

[19] P. Grassberger and R. Hegger, J. Chem. Phys. 102, 6881 (1995).

[20] P.G. Higgs and R.C. Ball, Macromolecules, 22, 2432 (1989).

[21] P. Grassberger and R. Hegger, Ann. Physik 4, 230 (1995).

[22] G.T. Barkema, U. Bastolla, and P. Grassberger, J. Stat. Phys. 90, 1311 (1998).

[23] A.L. Owczarek, T. Prellberg, and R. Brak, Phys. Rev. Lett. 70, 951 (1993).

[24] A.M. Ferrenberg and R.H. Swendsen, Phys. Rev. Lett. 61, 2635 (1988); 63, 1195 (1989).

[25] T. Prellberg and A. L. Owczarek, Phys. Rev. E 62, 3780 (2000); Europhys. Lett. 51, 602 (2000); Physica A 297, 275 (2001); cond-mat/0108390 (2001).

[26] V. Mehra and P. Grassberger, cond-mat/0107525 (2001)

[27] I. Carmesin and K. Kremer, Macromolecules 21, 2819 (1988).

[28] J.S. Liu, Monte Carlo Strategies in Scientific Computing, Springer Series in Statistics (Springer, New York 2001).

[29] P. Grassberger, J. Chem. Phys. 111, 440 (1999). 